\title{
Computerized Adaptive Testing for Public Opinion Surveys
}

\author{
Jacob M. Montgomery \\ Department of Political Science, Washington University in St. Louis, Campus Box 1063, \\ One Brookings Drive, St Louis, MO 63130-4899 \\ e-mail: jacob.montgomery@wustl.edu (corresponding author)
}

\author{
Josh Cutler \\ Department of Political Science, Duke University, 326 Perkins Library, Box 90204, Durham, \\ NC 27708
}

Edited by R. Michael Alvarez

\begin{abstract}
Survey researchers avoid using large multi-item scales to measure latent traits due to both the financial costs and the risk of driving up nonresponse rates. Typically, investigators select a subset of available scale items rather than asking the full battery. Reduced batteries, however, can sharply reduce measurement precision and introduce bias. In this article, we present computerized adaptive testing (CAT) as a method for minimizing the number of questions each respondent must answer while preserving measurement accuracy and precision. CAT algorithms respond to individuals' previous answers to select subsequent questions that most efficiently reveal respondents' positions on a latent dimension. We introduce the basic stages of a CAT algorithm and present the details for one approach to item selection appropriate for public opinion research. We then demonstrate the advantages of CAT via simulation and empirically comparing dynamic and static measures of political knowledge.
\end{abstract}

\section{Introduction}

Survey researchers often avoid using large multi-item scales to measure latent traits on surveys. In part, this reflects the high financial costs of long surveys. For most researchers, the primary cost associated with public opinion research is the per-question fee charged by survey firms. Thus, there are significant financial incentives for asking as few questions as possible when measuring any latent trait or attitude.

However, the desire to avoid large batteries also reflects the higher rate of attrition and nonresponse associated with lengthy and repetitive surveys. Numerous studies have shown that longer surveys are associated with higher rates of unit nonresponse (e.g., Heberlein and Baumgartner 1978; Yammarino, Skinner, and Childers 1991; Burchell and Marsh 1992; Crawford, Couper, and Lamias 2001; Galesic and Bosnjak 2009), a greater likelihood of halting an interview (e.g., Sheatsley 1983), and higher rates of item nonresponse (e.g., Anderson, Basilevsky, and Hum 1983). Moreover, lengthy repetitive surveys increase the burden on respondents who compensate by beginning to satisfice when selecting answers (Krosnick 1991, 1999), increasing use of “don’t know" responses (Krosnick et al. 2002), and providing generally less informative responses (Herzog and Bachman 1981).

To avoid long batteries, researchers typically select a subset of available items to include on a survey. For instance, in their study of the role of personality traits in determining political attitudes, Gerber et al. (2010) rely on the Ten Item Personality Inventory (TIPI), a much reduced alternative

\footnotetext{
Authors' note: We are grateful for helpful comments provided by Martin Elff, Sunshine Hillygus, Walter Mebane, Brendan Nyhan, and two anonymous reviewers. A previous version of this article was presented at the 2012 Annual Meeting of the Midwest Political Science Association, the 2012 Saint Louis Area Methods Meeting, and the 2012 Summer Methods Meeting. Supplementary materials for this article are available on the Political Analysis Web site.
} 
to the 44-item Big Five Inventory (BFI) (Gosling, Rentfrow, and Swann 2003). ${ }^{1}$ Likewise, the 2008-2009 American National Election Study panel included only two items adapted from the standard 18-item "need for cognition" scale (Cacioppo and Petty 1984). Indeed, developing reduced versions of larger scales constitutes a whole genre of research in the fields of psychology, consumer research, public opinion, and more. ${ }^{2}$ Yet, relying on reduced batteries can lower measurement precision and introduce bias - especially for individuals on the extreme ends of a latent scale.

In this article, we propose an alternative to the creation of static reduced scales. We apply computerized adaptive testing (CAT) to the field of public opinion surveys and introduce software that will aid researchers to more accurately measure important latent traits using a minimum number of question items. As its name suggests, CAT algorithms adapt dynamically to measure latent constructs while minimizing the number of questions each respondent must answer-similar to the functioning of the Graduate Record Examinations (GRE) prior to its revision in 2011. The method is an extension to item response theory (IRT), and like IRT, derives from the educational testing literature. Each question item is classified based on both its average level of "difficulty" (its position on the latent dimension) and its capacity to discriminate between respondents. CAT algorithms respond to individuals' prior answers by choosing subsequent questions that will place them on the latent dimension with maximum precision and a minimum number of questions.

In the rest of this article, we review the basic elements of CAT algorithms and explain in detail one approach to item selection and stopping rules appropriate for public opinion research. We then evaluate, both theoretically and empirically, the advantages of CAT relative to traditional static reduced batteries. First, we conduct a simulation study to show how CAT surveys can increase precision and reduce bias relative to static scales. Second, using a convenience sample of Amazon Mechanical Turk respondents, we conduct a survey experiment and compare the precision and accuracy of CAT surveys to static scales of political knowledge. In both theory and practice, when the two competing methods of measurement are compared on a common metric, CAT provides improved precision and reduced bias.

\title{
2 CAT and Traditional Dynamic Survey Techniques
}

\subsection{Computerized Adaptive Testing}

CAT algorithms are based on the notion that questions should be chosen for each respondent based, in part, on what we know about them from previous responses. Ignoring prior information leads us to waste valuable survey time asking respondents questions that are not revealing. Just as we would not ask a simple algebra question to assess the mathematical aptitude of a theoretical physicist, we should not ask survey respondents who have already correctly explained the reconciliation process in the US Senate whether they know how many branches are in the Federal Government. It is more informative to instead choose questions that reflect prior responses. In the language of educational testing:

\begin{abstract}
The basic notion of an adaptive test is to mimic automatically what a wise examiner would do. Specifically, if an examiner asked a question that turned out to be too difficult for the examinee, the next question asked would be considerably easier. This stems from the observation that we learn little about an individual's ability if we persist in asking questions that are far too difficult or far too easy for that individual. We learn the most when we accurately direct our questions at the same level as the examinee's proficiency (Wainer 1990, p. 10).
\end{abstract}

\subsection{Relationship to Traditional Dynamic Surveys}

In many ways, CAT is similar to branching survey techniques that have been used since the early days of public opinion surveys. For instance, the standard American National Elections Study

\footnotetext{
${ }^{1}$ The 44-item battery is itself an effort to develop a shorter (and publicly accessible) alternative to the 240-item NEO Personality Inventory-Revised.

${ }^{2}$ Just a few of the many recent examples of efforts to develop reduce scales in the literature include Podsakoff and MacKenzie (1994), Stanton et al. (2002), Russell et al. (2004), Richins (2004), Matthews, Kath, and Barnes-Farrell (2010), and Thompson (2012).
} 
measure of party identification asks whether respondents think of themselves as "a Republican, a Democrat, an Independent, or what?" Interviewers then ask Democratic identifiers whether they would call themselves a "strong Democrat." However, there is no purpose in asking self-identified Democrats if they would call themselves a "strong Republican." Based on prior beliefs about Democratic respondents and the nature of the "strong Republican" question, researchers know that little information will be gained by administering the "strong Republican" item to Democratic respondents.

CAT takes the logic behind these branching question formats and extends it to large survey batteries containing dozens (if not hundreds) of potential items - far more items than can easily be placed in a branching hierarchy. In essence, CAT algorithms use prior information about respondents and question items to more quickly and accurately place survey takers on some latent scale. Prior information about respondents is derived from their initial responses to items in the battery. Prior information about the items is derived from pre-testing the questionnaire. This establishes how specific questions relate to the latent scale of interest and provides the needed itemlevel parameters for the CAT algorithm to operate.

Beyond simple branching formats, CAT is also clearly related to computer-assisted interviewing techniques developed over two decades ago (Piazza, Sniderman, and Tetlock 1989; Sniderman et al. 1991). Like branching questionnaires, this approach allows for survey items to change based on a respondent's answers, experimental assignment, or any other criteria. Computer-assisted interviews can respond in complex ways during the interview process in a manner pre-specified by researchers.

\subsection{Relationship to Item Response Theory}

While related to computer-assisted surveys, CAT algorithms derive from an entirely different branch of research - educational testing. Computerized adaptive testing is itself an extension of IRT (Lord and Novick 1968; Lord 1980; Embretson and Reise 2000; Baker and Kim 2004), which has received considerable attention in recent years in political science (e.g., Clinton and Meirowitz 2001; Jackman 2001; Martin and Quinn 2002; Clinton and Meirowitz 2003; Clinton, Jackman, and Rivers 2004; Bafumi et al. 2005; Poole 2005; Bailey 2007; Treier and Jackman 2008; Treier and Hillygus 2009; Gillion 2012).

IRT is a general method for measuring latent traits using observed indicators, which are binary or ordinal in most political science applications. CAT takes the IRT framework and extends it to allow tests or surveys to be tailored to each individual respondent (Weiss 1982; Kingsbury and Weiss 1983; Weiss and Kingsbury 1984; Dodd et al. 1995). Items are selected based on respondents' answers to previously administered questions with the goal of choosing items that will be most revealing. Numerous studies have shown that CAT tests outperform traditional static tests of similar lengths (e.g., Weiss 1982; Weiss and Kingsbury 1984; Hol et al. 2007).

Since its initial inception in the late 1970s, CAT has been extended in a number of directions to allow for refinements such as content balancing (van der Linden 2010), informative priors (van der Linden 1999), response times (van der Linden 2008), multidimensionality (Segall 2010), nonparametric assumptions (Xu and Douglas 2006), and more (van der Linden and Pashley 2010). CAT is widely used in the fields of educational testing, psychology (e.g., Waller and Reise 1989; Forbey and Ben-Porath 2007), and (to a much lesser extent) marketing (Singh, Howell, and Rhoads 2007). However, CAT methods have rarely been applied in the measurement of public opinion. Moreover, we are aware of no instances of its use in published political science research. The purpose of this article, therefore, is to introduce the details of the CAT technique to the political science community, give guidance as to how it can be fruitfully incorporated into the study of public opinion, and provide a clear empirical demonstration of the significant advantages available from adopting and adapting this methodology.

\subsection{Relative Advantages of CAT Surveys}

CAT differs from more familiar methodologies, such as branching questionnaires, in two important ways. First, CAT is able to easily deal with much longer dynamic batteries than is feasible using 
traditional methods. For example, a dynamic battery that asks each respondent only 11 dichotomous items would require the pre-specification of over $2^{10}=1024$ possible branchings.

Second, CAT methods assume that the branching procedure is not something determined in advance by researchers. Rather, questions are chosen "online" as the survey is completed to maximize a pre-specified objective function. Item selection is therefore extremely formalized and directly embedded in the mathematics of the scaling procedure that translates survey responses into the latent trait space of interest. Indeed, the method assumes researchers are not interested in answers to the questions per se, but only in accurately estimating respondents' positions on the latent scale. One distinct advantage of CAT, therefore, is that choices about the ordering of questions need not be justified by researchers, as item selection is explicitly determined by available data in a theoretically motivated manner. The disadvantage is that researchers must gather calibration data in advance in order to specify the item parameters.

A hypothetical example can demonstrate the basic advantages of the CAT framework. Consider a survey battery that contains 40 items. As a running example, assume that these items are factual questions about the United States and international politics designed to measure political knowledge. Let us assume that there is space for five items and that all questions are dichotomous indicators (i.e., answers are either correct or incorrect).

One advantage of CAT is that it allows researchers to include a larger number of question combinations. On a large national survey, scholars typically choose just one subset of these items. However, in our example there are actually $\left(\begin{array}{c}40 \\ 5\end{array}\right)=658,008$ possible reduced batteries we could include. A five-item adaptive battery would allow us to include at least 16 question combinations, while a ten-item battery would include 512. ${ }^{3}$ Moreover, using informative priors, response times, and other refinements, CAT would allow us to theoretically include the entire collection of potential question combinations in the latent question-item space. ${ }^{4}$

A second and more important advantage is that CAT allows us to ask better question combinations chosen to reveal the most information about each respondent. With both adaptive and static batteries, we will be able to partition respondents into $2^{5}=32$ categories based on their response profiles. For fixed batteries, however, many of these potential response profiles are rarely (if ever) observed. Individuals who recognize the name of the Chief Justice of the Supreme Court are extremely unlikely not to recognize the Vice President. CAT, however, makes it far more likely that we will observe the full range of potential response profiles, as it chooses questions that respondents are expected to answer either correctly or incorrectly with roughly equal probability.

Finally, this added precision comes with no expense in terms of survey time, as each respondent is still asked only five questions. This advantage may be particularly important on national probability samples, where there are real and significant financial incentives to keep batteries short. It may also be important in situations where response rates are extremely sensitive to survey length such as interviews of political elites.

\section{Computerized Adaptive Testing Algorithms}

\subsection{Intuition}

Having reviewed the basic motivation for applying CAT algorithms, in this section we provide additional details about one implementation of the method appropriate for public opinion research. CAT is designed for application in the context of a large survey battery or psychometric scale whose validity has already been established. That is, the method assumes that there is actually some latent trait to be measured and that each of the candidate items are appropriate indicators of that trait.

\footnotetext{
${ }^{3}$ The response profiles can be modeled with a binary tree, where each node is a question and the branches correspond to correct/incorrect answers. The root node is the first question asked and the leaf nodes of this tree correspond to the number of response profiles we can obtain with the adaptive survey. In the five-item case, we have a tree of height 5 and thus $2^{4}$ possible response profiles. In the ten-item case we have $2^{9}$.

${ }^{4} \mathrm{We}$ focus in this article only on the simplest version of CAT. Implementation and analysis of adaptive algorithms that leverage prior information and response times for survey research remains a task for future research.
} 
Potential applications in political science include large psychometric batteries (e.g., Gosling, Rentfrow, and Swann 2003), batteries on issue positions designed to place respondents into an ideological space (Treier and Hillygus 2009; Bafumi and Herron 2010), or a listing of forms of political participation respondents may have engaged in over the past year (Gillion 2012).

CAT may be particularly useful in instances where researchers care about individuals who are "extreme" along some dimension of interest. This might include political activism (e.g., Verba et al. 1995), racial attitudes (e.g., Feldman and Huddy 2005), political knowledge or sophistication (e.g., Zaller 1992), or political ideology (e.g., Bafumi and Herron 2010).

CAT is a method for taking a large population of potential items and selecting among them to efficiently place respondents on some latent scale such as ideology, political knowledge, or activism. Roughly speaking, the algorithm chooses items that are most likely to produce the most precise and accurate estimate of respondents' position on a latent factor.

Ceteris paribus, CAT achieves this goal by choosing items with larger discrimination parameters. That is, it prefers items that are most revealing about respondents once they have answered. Second, ceteris paribus, CAT will choose items whose difficulty parameters are "close" to the current estimate of the respondent's ability parameter. The algorithm prefers questions that it estimates the survey taker has a roughly equal chance of answering correctly and incorrectly. Additional intuition regarding how CAT chooses among available items is provided in our simulated example below.

\subsection{Algorithm Essentials}

A general overview of a basic CAT algorithm is fairly straightforward, although there are a wide array of increasingly complex implementations in the literature (van der Linden and Pashley 2010). The essential elements of computerized adaptive tests are shown in Table 1 (Segall 2005, 4).

First, estimates $\left(\hat{\theta}_{j}\right)$ are generated for each respondent's position on the latent scale of interest $\left(\theta_{j}\right)$. Before the first item is administered, this estimate is based on our prior assumptions about $\theta_{j}$. One option is to assume a common prior for all respondent's, $\theta_{j} \sim \pi(\theta)$. An alternative is to use previously collected data points, $\mathbf{y}_{j}$, to specify an informative prior, $\theta_{j} \sim \pi\left(\theta_{j} \mid \mathbf{y}_{j}\right)$ (van der Linden 1999). For example, when administering a battery measuring political ideology it may be appropriate to assume that strong Democrats are more liberal than strong Republicans. In either case, after the initial item in the CAT battery is administered, $\mathbf{y}_{j}$ will include responses to items that have already been administered and answered.

Second, the subsequent question item is selected out of the available battery. CAT chooses the item that optimizes some pre-specified objective function. Multiple criteria appear in the literature, including maximum Fisher information (MFI), maximum likelihood weighted information (MLWI), maximum expected information (MEI), minimum expected posterior variance (MEPV), and maximum expected posterior weighted information (MEPWI) (Choi and Swartz $2009,421) .{ }^{5}$ It is also possible to choose constrained optimization approaches to ensure, for instance, that scales balance items that are negatively and positively worded.

In general, these criteria aim to choose items that will result in accurate and precise measures. Moreover, all of these item-selection criteria lead to similar results after a modestly large number of items (i.e., $n \geq 30$ ). However, there are significant differences in measurement quality when the number of items that can be asked is more limited (van der Linden 1998). In this article, we have chosen to focus on the MEPV criterion because (1) we feel it is the most intuitive and mathematically motivated approach, and (2) it is among the criteria that previous research has shown performs well with a small number of questions.

The third stage of the algorithm is to administer the chosen item and record the response. Fourth, the algorithm checks some stopping rule. In most survey settings, the stopping rule is

\footnotetext{
${ }^{5} \mathrm{An}$ overview of the most common item selection criteria for dichotomous indicators is discussed in van der Linden (1998) and van der Linden and Pashley (2010). An excellent analysis of potential selection criteria for polytomous items is available in Choi and Swartz (2009).
} 
Table 1 Basic elements of computerized adaptive testing batteries

\begin{tabular}{|c|c|c|}
\hline Stage & Purpose & Description \\
\hline 1 & $\begin{array}{l}\text { Estimate } \\
\text { respondents' positions }\end{array}$ & $\begin{array}{l}\text { A provisional trait estimate, } \hat{\theta}_{j} \text {, is created based on first } i \text { responses. If } \\
\text { no items have been given, the estimate is based on prior information. }\end{array}$ \\
\hline 2 & Item selection & $\begin{array}{l}\text { The item that optimizes some objective function is chosen. In our } \\
\text { examples below, CAT chooses items that minimize expected posterior } \\
\text { variance. }\end{array}$ \\
\hline 3 & Administer item & \\
\hline 4 & Check stopping rule & $\begin{array}{l}\text { Pre-defined stopping rules may include reducing posterior variance, } \\
\operatorname{Var}\left(\hat{\theta}_{j}\right) \text {, below a certain threshold or reaching some maximum time } \\
\text { allotment for the battery. }\end{array}$ \\
\hline $5 a$ & Repeat steps $1-4$ & If the stopping rule has not been reached, new items are administered. \\
\hline $5 b$ & $\begin{array}{l}\text { Return final trait } \\
\text { estimate }\end{array}$ & $\begin{array}{l}\text { If the stopping rule has been reached, a final estimate for } \hat{\theta}_{j} \text { is } \\
\text { calculated. }\end{array}$ \\
\hline
\end{tabular}

likely to be that the number of items asked of the respondent has reached some maximum value. In these fixed-length CAT algorithms, all respondents will be asked the same number of questions. However, it is also possible that researchers wish to measure some trait up to a specific level of precision regardless of the number of items that are asked. In these variable-length CAT algorithms, items may be administered until this threshold is reached.

Finally, if the stopping rule has not been reached, the algorithm will repeat. Once the stopping criterion has been met, the algorithm produces final estimates of $\hat{\theta}_{j}$ and terminates.

\subsection{Outline of the General Model for Dichotomous Indicators}

As discussed above, there are numerous variants of CAT for both dichotomous and polytomous indicators. Rather than attempting to summarize all of these approaches here, we will focus on the particular specification we use in our examples below. We will also restrict ourselves to the dichotomous case, which is both more intuitive and more familiar to a political science audience due to its wide use in roll call analyses (c.f., Clinton, Jackman, and Rivers 2004; Bafumi et al. 2005).

We use a two-parameter logistic model, where $y_{i j}$ is the observed outcome (correct/incorrect or yes/no) for item $i \in[1, n]$ and person $j \in[1, J]$. The model assumes that the probability of a correct response for individual $j$ is

$$
p_{i}\left(\theta_{j}\right) \equiv \operatorname{Pr}\left(y_{i j}=1 \mid \theta_{j}\right)=\frac{\exp \left(D a_{i}\left(\theta_{j}-b_{i}\right)\right)}{1+\exp \left(D a_{i}\left(\theta_{j}-b_{i}\right)\right)},
$$

where $D=1$ for a logistic model and $D=1.702$ for an approximation of the probit model.

We assume that the item-level parameters $\left(a_{i}, b_{i}\right)$ have already been estimated using some previously collected data, which we term the calibration sample below. These parameters are typically termed the discrimination and difficulty parameters, respectively. For CAT, we assume that these are known quantities and our interest is only in estimating the ability parameter, $\theta_{j}$, for some new respondent.

The prior distribution for $\theta_{j}$ will be

$$
\pi\left(\theta_{j}\right) \sim N\left(\mu_{\theta}, \frac{1}{\tau_{\theta}}\right)
$$

where $\tau_{\theta}$ denotes the precision of the distribution (the inverse of the variance). In our examples below, we set $\mu_{\theta}=0$ and $\tau_{\theta}=0.6$, a fairly diffuse (though proper) prior. ${ }^{6}$

\footnotetext{
${ }^{6}$ In numerous simulation experiments, we found this setting to be ideal for the purposes of small batteries. Stronger priors (e.g., $\tau_{\theta}=1$ ) result in item selection being dominated by the prior, which is only overcome after many items are
} 
Letting $q_{i}\left(\theta_{j}\right)=1-p_{i}\left(\theta_{j}\right)$, the likelihood function associated with the responses to the first $k-1$ items under a local independence assumption is

$$
L\left(\theta_{j} \mid \mathbf{y}_{k-1, j}\right)=\prod_{i=1}^{k-1} p_{i}\left(\theta_{j}\right)^{y_{i j}} q_{i}\left(\theta_{j}\right)^{\left(1-y_{i j}\right)}
$$

\subsection{Calculating Skill Parameter}

We present one of the most prominent methods for calculating respondent-level positions on a latent scale. ${ }^{7}$

The expected a posteriori (EAP) estimate of individual $j$ 's position on the latent scale is calculated as

$$
\hat{\theta}_{j}^{\text {(EAP) }} \equiv E\left(\theta_{j} \mid \mathbf{y}_{k-1, j}\right)=\frac{\int \theta_{j} \pi\left(\theta_{j}\right) L\left(\theta_{j} \mid \mathbf{y}_{k-1, j}\right) d \theta_{j}}{\int \pi\left(\theta_{j}\right) L\left(\theta_{j} \mid \mathbf{y}_{k-1, j}\right) d \theta_{j}} .
$$

Neither of these integrals can be analytically derived. However, using numerical methods, we can approximate these quantities with sufficient precision. ${ }^{8}$

\subsection{Item Selection}

We use the MEPV criterion to select items. This requires that we first estimate the posterior variance associated with a correct $\left(y_{k j}^{*}=1\right)$ and incorrect $\left(y_{k j}^{*}=0\right)$ response for all remaining items and multiply by the probability of observing these outcomes conditioned on the current estimate of $\theta_{j}$.

We first estimate $P\left(y_{k j}^{*}=1 \mid \mathbf{y}_{k-1, j}\right)=1-P\left(y_{k j}^{*}=0 \mid \mathbf{y}_{k-1, j}\right)$, where $\mathbf{y}_{k-1, j}=y_{1, j}, \ldots, y_{k-1, j}$. This is done by simply entering the current value of $\hat{\theta}_{j}$ into equation (1) for item $k$. We then calculate

$$
\hat{\theta}_{j}^{(\mathrm{EAP}) *} \equiv E\left(\theta_{j} \mid \mathbf{y}_{k-1, j}, y_{k j}^{*}\right),
$$

which is the estimator conditioned on the potential response for the candidate item $k$, denoted $y_{k j}^{*}$. The posterior variance for each possible response to each potential item is

$$
\operatorname{Var}\left(\theta_{j} \mid \mathbf{y}_{k-1, j}, y_{k j}^{*}\right)=E\left(\left(\theta_{j}-\hat{\theta}_{j}^{(\mathrm{EAP}) *}\right)^{2} \mid \mathbf{y}_{k-1, j}, y_{k j}^{*}\right)
$$

\footnotetext{
asked. Weaker priors (e.g., $\tau_{\theta}=0.01$ ) result in extreme and uninformative items being selected when $n$ is small. Note that we only set this prior during item selection. For the final estimation of $\hat{\theta}_{j}$, we return to the same $\tau_{\theta}=1$ prior used for parameter estimation on the calibration data.

${ }^{7} \mathrm{~A}$ second common technique is to estimate the maximum a posteriori (MAP), which is found by estimating the root of the first derivative of the $\log$ posterior (equation 3 ):

$$
\frac{\partial \log L\left(\theta_{j} \mid \mathbf{y}_{k-1, j}\right)}{\partial \theta_{j}}=\sum_{i=1}^{k-1} \frac{p_{i}^{*}\left(\theta_{j}\right)\left(y_{i j}-p_{i}\left(\theta_{j}\right)\right)}{p_{i}\left(\theta_{j}\right) q_{i}\left(\theta_{j}\right)},
$$

where

$$
p_{i}^{*}\left(\theta_{j}\right) \equiv \frac{\partial p_{i}\left(\theta_{j}\right)}{\partial \theta_{j}}=D a_{i}\left(d_{i}-c_{i}\right) \frac{\exp \left(D a_{i}\left(\theta_{j}-b_{i}\right)\right)}{\left(1+\exp \left(D a_{i}\left(\theta_{j}-b_{i}\right)\right)\right)^{2}}
$$

${ }^{8}$ In most IRT models in the political science literature, these estimates are done using Markov chain Monte Carlo (MCMC) simulation. However, since these are both one-dimensional integrals, we deem such an approach unnecessary. In the current version of our software, we use an approximation through the integrate.xy() function in the sfsmisc package. This is appropriate as the model is identified by fixing the distribution of "ability" parameters to cluster relatively tightly near zero, making numerical integration less likely to produce a significant error. Alternative parameterizations that allow for a broader possible range for $\theta_{j}$ may require alternative implementations.
} 


$$
=\frac{\int\left(\theta_{j}-\hat{\theta}_{j}^{(\mathrm{EAP}) *}\right)^{2} \pi\left(\theta_{j}\right) L\left(\theta_{j} \mid \mathbf{y}_{k-1, j}, y_{k j}^{*}\right) d \theta_{j}}{\int \pi\left(\theta_{j}\right) L\left(\theta_{j} \mid \mathbf{y}_{k-1, j}, y_{k j}^{*}\right) d \theta_{j}},
$$

which is estimated via numerical integration as above. Equation (7) represents the posterior variance we will observe if the algorithm administers item $k$ to respondent $j$ and the answer given is $y_{k j}^{*}$. According to the MEPV criterion, the item chosen will minimize the value of

$$
\begin{aligned}
& P\left(y_{k j}^{*}=1 \mid \mathbf{y}_{k-1, j}\right) \operatorname{Var}\left(\theta_{j} \mid \mathbf{y}_{k-1, j}, y_{k j}^{*}=1\right)+ \\
& P\left(y_{k j}^{*}=0 \mid \mathbf{y}_{k-1, j}\right) \operatorname{Var}\left(\theta_{j} \mid \mathbf{y}_{k-1, j}, y_{k j}^{*}=0\right) .
\end{aligned}
$$

\subsection{Stopping Criteria}

In our examples below, the algorithm stops offering items when the number of questions reaches a pre-specified threshold $n_{\max }$. An alternative, however, is to stop when the posterior precision, $V\left(\theta_{j} \mid \mathbf{y}_{j}\right)^{-1}$, rises above some pre-specified level $\tau_{\theta}^{\text {stop }}$. This option might be particularly useful when researchers seek homoscedastic measurement variance. ${ }^{9}$

\section{Simulation and Illustration}

In this section, we seek to demonstrate the potential advantages of CAT through a simulation study. These simulations represent circumstances that are as ideal as can be expected in a survey setting. The item pool consists of 60 items, and the response probabilities align exactly with the twoparameter logistic model in equation (1). The discrimination parameters are drawn from $a_{i} \sim \operatorname{Gamma}(50,25)$, and the difficulty parameters $\left(b_{i}\right)$ are spaced equidistantly on the interval $[-3,3]$. In essence, the simulation assumes that we have 60 items that load strongly on the underlying latent dimension $(\bar{a}=2)$, with item difficulty parameters spanning the range of likely ability parameters.

\subsection{Illustrative Simulated Example}

We begin by comparing how fixed and dynamic batteries of identical length estimate the position of a single exemplar individual. The focus here is to illustrate why, under some circumstances, CAT can provide less biased and more precise estimates of $\theta_{j}$.

Reduced scales, both in our simulations and in the real world, are typically chosen to optimize measurement precision for respondents near the center of the latent distribution. For instance, in developing a reduced scale measuring aspects of personality, Gosling, Rentfrow, and Swann (2003, 508) state that "where possible we selected items that were not evaluatively extreme." This is because it is items that reveal the most information about individuals at the center of the distribution that will minimize total absolute bias and error. Put simply, most respondents are located in the middle of the distribution, so it makes sense to choose items biased towards the center. However, this strategy often results in imprecise and even biased estimates of respondents located toward the extreme ends of the latent scale.

More fundamentally, reduced scales almost inevitably include some questions that are either too "easy" or too "hard" for a given respondent. This results in inefficiencies, since questions are administered to respondents that provide no additional insight as to their true position on the latent scale.

This is illustrated in Fig. 1, which shows the item characteristic curves (ICC) for a fixed (left panels) ${ }^{10}$ and dynamic (right panels) battery administered to a single individual whose true position

\footnotetext{
${ }^{9}$ Note that one is not guaranteed the same level of variance in the estimate of $\hat{\theta}$ for each respondent when simply asking a fixed number of questions. Using this alternative stopping rule can help ensure that the estimates have equal variance and are more amenable to regression analysis.

${ }^{10}$ Specifically, the battery includes Items $10,20,30,40$, and 50 . As items are spread equidistantly, this represents a highquality fixed battery.
} 

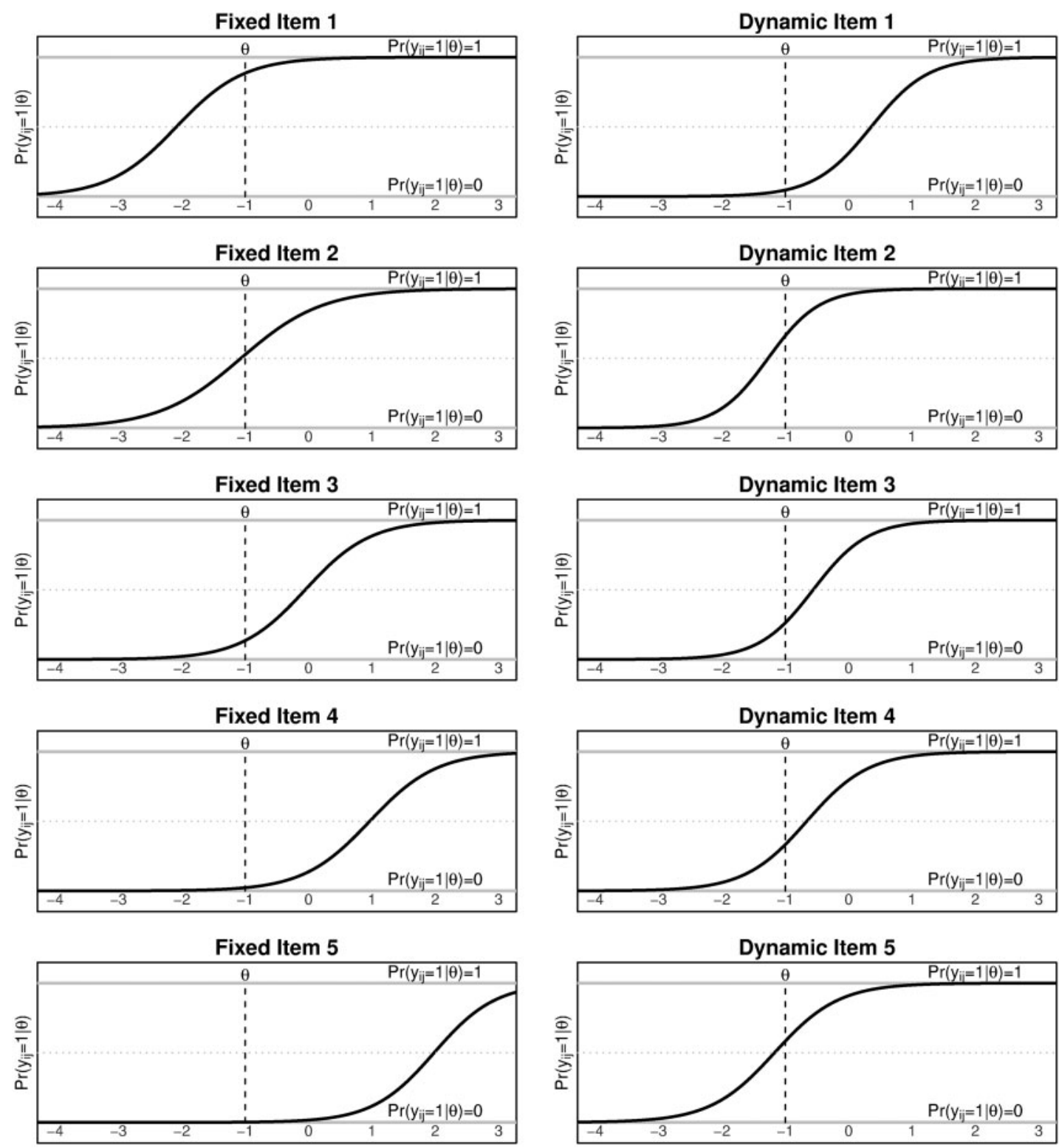

Fig. 1 Item characteristic curves for a fixed and dynamic five-item battery. The left panels show the item characteristic curves for the five items in the static scale. The right panel shows these same curves for the items as chosen by the CAT algorithm. Note that the CAT algorithm chooses items that the respondent, whose position is indicated with a vertical line, has a significant probability of answering either correctly or incorrectly. For the static battery, the respondent is extremely unlikely to answer Items 4 and 5 correctly.

on the latent scale is indicated by the vertical dashed line. ${ }^{11}$ Item characteristic curves show the predicted probability of answering a question affirmatively (i.e., getting the question "right") for individuals of varying skill levels $\left(\theta_{j}\right)$. Thus, the horizontal axis shows the different potential values of $\theta_{j}$, while the vertical axis shows the probability of answering affirmatively for each value of $\theta_{j}$.

\footnotetext{
${ }^{11}$ Recall that that MEPV selection criteria choose the item that minimizes the function shown in equation (8). The selected items will generally have (1) large discrimination parameters and (2) difficulty parameters located near the algorithm's current estimate of $\hat{\theta}$. If most items have fairly similar discrimination parameters, the latter criteria tends to dominate. Note that, even in a simulated example where all items perform well, there is not always a strictly smooth relationship between an item's difficulty and its EPV due to heterogeneity in discrimination parameters.
} 
Note especially the ICCs shown in the four bottom-most panels of Fig. 1. The ICC curve for the fixed battery indicates that the respondent, whose position is at -1 on the latent scale, will almost certainly not answer either Item 4 or Item 5 in the fixed battery affirmatively. The predicted probability of doing so is nearly zero. Thus, as we show below, no additional information about the respondent is gained by asking these questions. On the other hand, the items chosen by the CAT battery are all such that the respondent has a significant probability of answering in either direction. This suggests that we will learn more about the respondent as each additional question in the CAT battery is asked and answered.

This intuition is confirmed by looking at the estimated posteriors for $\theta_{j}$ shown in Fig. 2. The figure shows the true value $\left(\theta_{j}\right)$ and the posterior estimates of $\left(\hat{\theta}_{j}\right)$ given responses to all previously administered items for both the static (left panels) and dynamic (right panels) batteries. ${ }^{12}$

There are two aspects of Fig. 2 we wish to emphasize. First, the final estimate for the static battery $\left(\hat{\theta}_{j}=-0.53\right)$ is relatively inaccurate compared to the final estimate of the dynamic battery $\left(\hat{\theta}_{j}=-0.89\right)$. Second, neither the precision nor the accuracy of the estimate are improved after the administration of Items 4 and 5 in the static scale. That is, over $40 \%$ of the battery provides almost no additional information about this respondent. In contrast, the right panels of Fig. 2 show the posteriors as determined by items chosen by the CAT algorithm. As can be seen, the estimate of $\theta_{j}$ is more accurate and far more precise. Moreover, the posterior continues to converge toward the true value of $\theta_{j}$ after each question is administered and answered.

\subsection{Systematic Simulation}

While the results in Figs. 1 and 2 are illustrative, they do not provide systematic evidence in favor of CAT. We therefore seek to generalize these results across a broader range of values of $\theta$. Figure 3 shows the squared error ${ }^{13}$ for the dynamic (gray) and static (black) batteries of various lengths. ${ }^{14}$ The upper left panel shows the results for the case when the number of items is three, and the remaining panels show results when the battery length is five, seven, and ten items respectively. ${ }^{15}$ These estimates were generated for 1000 simulated respondents distributed equidistantly on the interval $[-3,3]$. This provides a fairly precise understanding for how the CAT algorithms perform across the possible range of $\theta_{j}$. The two sets of curves show the squared error (the vertical axis) that would result from the administration of static and dynamic batteries for individuals with differing positions on the underlying latent scale (the horizontal axis).

There are three aspects of the results shown in Fig. 3 that are helpful for understanding the advantages of CAT. First, across the entirety of the range of values of $\theta_{j}$, the dynamic survey results in a lower squared error. That is, for survey batteries of a similar length, a dynamic survey provides more accurate and more precise estimates of the latent trait. ${ }^{16}$

Second, the relative advantage of CAT diminishes somewhat toward the middle of the range of values for $\theta_{j}$. For example, in the lower left panel of Fig. 3 the static scale performs more equally with the dynamic scale for values of $\theta_{j}$ near zero. This is an expected finding that replicates results from previous simulation studies (e.g., van der Linden 1998). It indicates the degree to which fixed batteries are optimized to accurately measure individuals near the center of the distribution. ${ }^{17}$

\footnotetext{
${ }^{12}$ For illustrative purposes, we assume that responses are deterministic. That is, respondents always answer affirmatively (correctly) when the predicted probability is greater than 0.5 .

${ }^{13}$ Squared error is defined as $\operatorname{Var}\left(\hat{\theta}_{j}^{(\mathrm{EAP})}\right)+\left(\theta_{j}-\hat{\theta}_{j}^{\mathrm{EAP})}\right)^{2}$.

${ }^{14}$ The static batteries are: Items $15,30,45$ for $n=3$; Items $10,20,30,40,50$ for $n=5$; Items $9,16,23,30,37,44,51$ for $n=7$; and Items $3,9,15,21,27,33,39,45,51,57$ for $n=10$. Alternative methods for selecting fixed batteries make little difference in the substantive conclusions of these simulations.

${ }^{15}$ The stopping rule chosen for this and the empirical example is based on our belief that the primary constraint for public opinion researchers is time. While another rule, such as a measurement precision threshold, could have been used, this did not seem realistic given the budget and time constraints of most public opinion surveys.

${ }^{16}$ Indeed, examining the bias alone shows that CAT also provides lower bias for nearly all values of $\theta_{j}$. Improvement in unbiasedness is especially large in the extreme range of $\theta_{j}$ (results not shown).

${ }^{17}$ It makes sense that static scales are aimed at individuals in the "middle" of the latent space, as this is where most individuals will be located. However, the advantage of CAT is that the algorithm will tailor the battery to efficiently
} 

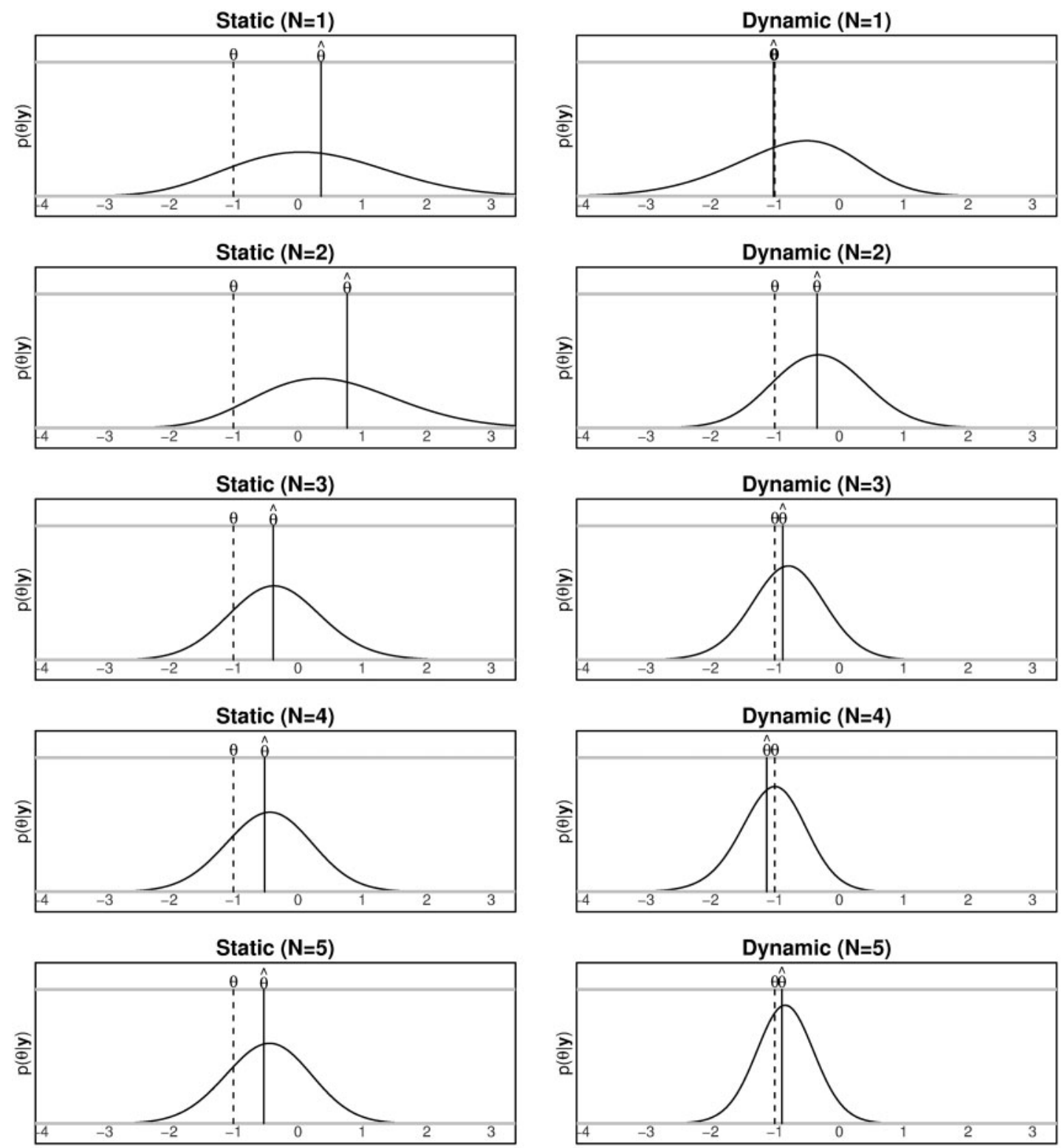

Fig. 2 Exemplar posterior estimates for a five-item static and dynamic battery. The left panels show the posterior estimates for the position of a single individual after each of five items in a fixed scale. The right panels show the posterior estimate after items as chosen by a dynamic scale. The true value $(\theta)$ and estimate $(\hat{\theta})$ are indicated with the dashed and solid vertical lines, respectively. Note that the posterior for the dynamic scale continues to converge to the true value of $\theta$ for all five items, while little additional information is garnered from the administration of the final two items in the fixed sale.

Finally, Fig. 3 shows visually how CAT divides respondents into a larger number of categories or "bins" than traditional static scales. By asking questions each respondent is more likely to answer right or wrong, CAT increases the likelihood of observing a positive or negative response to each question. A clear example of this is visible in the upper-left panel of Fig. 3. With three questions, we can potentially observe $2^{3}=8$ response profiles. Indeed, we can see that the CAT

estimate a latent trait regardless of the respondents' positions in the latent space. As we show, this improves measurement for all individuals, but the comparative advantage is greatest for more extreme individuals. 


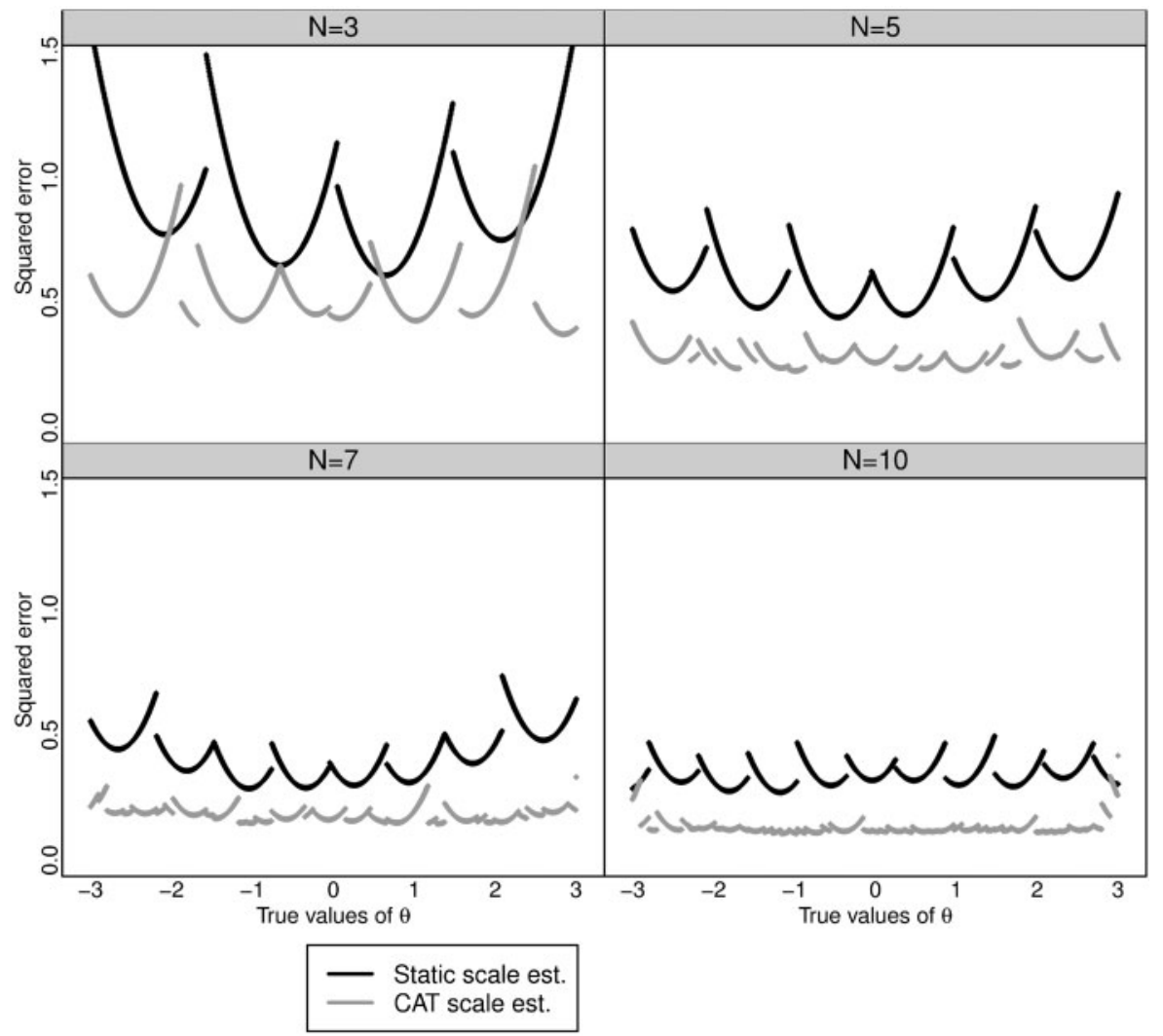

Fig. 3 Squared error for dynamic and static scales of four different lengths for simulated respondents. The curves show the squared error, defined as $\operatorname{Var}\left(\hat{\theta}_{j}^{(\mathrm{EAP})}\right)+\left(\theta_{j}-\hat{\theta}_{j}^{(\mathrm{EAP})}\right)^{2}$, for simulated individuals with differing values on the latent scale $(\theta)$. The point $\{0,1\}$ is associated with a squared error of 1 for an individual whose true position on the latent scale is $\theta_{j}=0$. The dark curves show the estimated squared error that results with administering a static scale, while the lighter curves show the squared error associated with a CAT scale of identical length $(n=3,5,7$, and 10$)$.

scale divides respondents into exactly eight categories, as there are eight "U" shaped bins visible. However, with a static scale we observe only four. ${ }^{18}$ Increasing the number of observed response profiles improves both measurement accuracy and precision.

\section{Empirical Application: Political Knowledge}

The simulation results in Section 4 show the advantages of CAT relative to a static scale theoretically. In this section, we provide an empirical application of CAT to the domain of political knowledge (sometimes termed political sophistication). This example demonstrates the superiority of CAT relative to static scales for accurately and precisely measuring important concepts to political science.

Although scholars have developed a number of measures for knowledge and sophistication (e.g., Luskin 1987; Delli Carpini and Keeter 1993), one of the most widely used methods for survey researchers is to ask questions measuring knowledge of basic facts about American politics, public officials, and current events. Since 1986 the American National Election Study (ANES) has asked respondents to identify the "job or political office" of officials such as the Vice President, the Speaker of the House, the Chief Justice of the United States Supreme Court, and the Prime

\footnotetext{
${ }^{18}$ Recall that in these simulations answers are deterministic (see Footnote 12). In a static scale, therefore, there will always be $n+1$ bins while there will be $2^{n}$ bins for the dynamic batteries.
} 
Minister of the United Kingdom (DeBell 2012). While these four items allow open-ended responses, other commonly used items are similar to standard multiple choice questions used in educational testing. For instance, the 1992 ANES asked respondents:

Who has the final responsibility to decide if a law is or is not constitutional ... is it the President, the Congress, the Supreme Court, or don't you know?

While this method of measuring political knowledge is used widely in public opinion research (e.g., Delli Carpini and Keeter 1993, 1996; Gomez and Wilson 2001; Barabas 2002; Brewer 2003), it has also been extensively criticized (c.f., Mondak 2001; Mondak and Davis 2001; Mondak and Anderson 2004; Lupia 2006, 2008; Prior and Lupia 2008; Gibson and Caldeira 2009; Luskin and Bullock 2011; DeBell 2012; Prior 2012). The coding of the open-ended responses is of questionable reliability, and on occasion entirely incorrect. ${ }^{19}$ Some have argued that the heavy emphasis on identifying prominent individuals does not seem a valid indicator of "political sophistication," or the ability to engage coherently in the political system (Lupia 2006). Finally, the items themselves do not seem appropriately chosen to acquire useful information about most respondents. In the 2008 ANES, 4\% of respondents correctly identified the office held by John Roberts, 5\% of respondents identified Gordon Brown, 37\% identified Nancy Pelosi, and 73\% correctly identified Dick Cheney (DeBell 2012). ${ }^{20}$

In this section, therefore, we apply a much larger collection of closed form (i.e., multiple choice) questions designed to measure much broader areas of political knowledge necessary for successfully engaging in the political system. In addition, the questions were designed to supply sufficient variation in difficulty.

\subsection{Model Calibration}

We developed a battery of 70 multiple-choice knowledge questions, six of which were dropped due to poor performance. These items were largely drawn from questions used previously in national samples (e.g., Luskin and Bullock 2011). The remaining 64 items, which measure knowledge in areas including the legislative process, interest groups, foreign affairs, and constitutional rights, are listed in our online supplementary materials.

We administered the battery to 810 respondents based in the United States and over the age of 18 years. Respondents were recruited through Amazon Mechanical Turk. ${ }^{21}$ This sample primarily serves to calibrate the model and allows us to estimate appropriate difficulty and discrimination parameters. Ideally, this calibration would be done on a nationally representative sample, which would give more meaningful estimates that could be used by researchers in future studies. ${ }^{22}$ However, this convenience sample serves the more limited purpose of illustrating the usefulness of the CAT method.

\footnotetext{
${ }^{19}$ DeBell (2012) notes that in 2004 identifying Tony Blair as the "Prime Minister of the United Kingdom" was coded as an incorrect response.

${ }^{20}$ These percentages change depending on how "correct" responses are coded. In addition, these numbers appear to fluctuate wildly from year to year. In 2004, 9.3\% identified Dennis Hastert, 28\% identified William Rehnquist, $62 \%$ identified Tony Blair, and 84\% identified Dick Cheney (Gibson and Caldeira 2009).

${ }^{21}$ Berinsky, Huber, and Lenz (2012) provide a detailed analysis of Mechanical Turk participants. Among their findings, which is reflected in our results below, is that Turk participants have much higher levels of political knowledge than the general US population. Although this limits the degree to which the item calibrations from this sample can be used for CAT algorithms administered to other populations, it does not alter the nature of our findings about the relative advantages of the CAT approach itself. Since the calibration and test samples are drawn from the same population, the underlying distribution of political knowledge can be assumed to be similar. It is the similarity in the distribution of political knowledge in the two samples that allows the CAT algorithm to outperform static batteries in the analyses below. To the degree that we might be concerned that this assumption is not correct as a result of our nonrandom sampling procedure, this fact only makes the significant improvement in measurement quality we show below more remarkable. In general, the more accurate the calibration of the item parameters, the better the performance of CAT will be relative to any given static scale.

${ }^{22}$ Using a national sample would allow us to say that difficulty parameters would, for instance, indicate the degree to which an average American can correctly answer a specific question. Researchers should avoid using convenience samples to calibrate the CAT algorithm unless they are comfortable that the distribution of the calibration sample is "representative" of the overall population of interest on the given latent trait.
} 
Table 2 Item-level parameters estimated from calibration sample

\begin{tabular}{|c|c|c|c|c|c|}
\hline Question & Difficulty & Discrimination & Question & Difficulty & Discrimination \\
\hline 1 & -2.57 & 1.72 & 33 & -0.61 & 0.97 \\
\hline 2 & -2.50 & 1.36 & 34 & -0.54 & 0.71 \\
\hline $3^{\mathrm{a}}$ & -2.34 & 2.12 & 35 & -0.51 & 0.70 \\
\hline 4 & -2.17 & 0.51 & 36 & -0.43 & 1.33 \\
\hline 5 & -2.17 & 1.77 & 37 & -0.41 & 1.35 \\
\hline 6 & -2.03 & 1.18 & 38 & -0.33 & 1.47 \\
\hline 7 & -2.00 & 1.00 & 39 & -0.28 & 1.12 \\
\hline 8 & -1.97 & 1.55 & 40 & -0.26 & 1.05 \\
\hline 9 & -1.89 & 1.23 & 41 & -0.18 & 2.06 \\
\hline 10 & -1.81 & 1.57 & 42 & -0.13 & 1.39 \\
\hline 11 & -1.62 & 1.84 & $43^{\mathrm{a}}$ & -0.07 & 1.65 \\
\hline 12 & -1.60 & 1.85 & 44 & -0.04 & 1.60 \\
\hline $13^{\mathrm{a}}$ & -1.59 & 1.63 & 45 & 0.12 & 0.92 \\
\hline 14 & -1.59 & 2.18 & 46 & 0.15 & 1.27 \\
\hline 15 & -1.57 & 1.22 & 47 & 0.17 & 1.57 \\
\hline 16 & -1.51 & 1.58 & 48 & 0.18 & 1.34 \\
\hline 17 & -1.46 & 1.38 & $49^{\mathrm{a}}$ & 0.20 & 1.65 \\
\hline 18 & -1.42 & 2.03 & 50 & 0.31 & 1.51 \\
\hline 19 & -1.40 & 1.61 & 51 & 0.35 & 1.23 \\
\hline 20 & -1.30 & 1.78 & 52 & 0.39 & 1.44 \\
\hline 21 & -1.25 & 0.98 & 53 & 0.40 & 0.89 \\
\hline $22^{\mathrm{a}}$ & -1.17 & 1.80 & 54 & 0.42 & 0.86 \\
\hline 23 & -1.16 & 0.67 & 55 & 0.43 & 1.05 \\
\hline 24 & -1.01 & 0.89 & $56^{\mathrm{a}}$ & 0.53 & 1.45 \\
\hline 25 & -0.98 & 1.51 & 57 & 0.60 & 1.25 \\
\hline 26 & -0.95 & 0.66 & 58 & 0.60 & 0.92 \\
\hline $27^{\mathrm{a}}$ & -0.85 & 1.71 & $59^{\mathrm{a}}$ & 1.14 & 0.81 \\
\hline 28 & -0.84 & 1.00 & 60 & 1.34 & 0.66 \\
\hline 29 & -0.72 & 1.41 & 61 & 2.50 & 0.61 \\
\hline 30 & -0.71 & 1.95 & $62^{\mathrm{a}}$ & 2.73 & 1.11 \\
\hline $31^{\mathrm{a}}$ & -0.69 & 2.04 & 63 & 2.98 & 0.83 \\
\hline 32 & -0.64 & 1.63 & 64 & 4.25 & 0.53 \\
\hline
\end{tabular}

a Item included in 10-item fixed scale. The model was estimated using the $1 \mathrm{tm}$ () command in the $1 \mathrm{tm}$ package in Rv2.15. Standard errors are suppressed for clarity. $n=810$.

Table 2 presents the item-level parameters associated with each of the questions in our battery. Items are ordered according to their difficulty parameters. The easiest question (the item with the lowest difficulty parameter) identified by this sample is Question 1, "How long is one term for the President of the United States? (a) Eight years, (b) Six years, (c) Four years, (d) Two years." The hardest question (the item with the largest difficulty parameter) is Question 64: "On which of the following does the US federal government spend the most money each year? (a) Education, (b) Medicare, (c) Interest on the national debt, (d) National defense." 23 Broadly speaking, respondents ordered questions in difficulty as we would expect. While there are available items for all levels of difficulty, there is a skew toward the lower end of the difficulty spectrum. This is unsurprising as we relied on items originally designed for national probability samples.

\footnotetext{
${ }^{23}$ Response options were randomized for all respondents except where responses had a clear numerical ordering. In addition, respondents were always allowed to answer that they did not know the answer or to simply skip the question (after a five-second delay). All "Don't know" and skipped questions were coded as incorrect responses.
} 


\subsection{Empirical Comparison with Reduced Scales}

To assess the efficacy of CAT techniques outside the calibration sample, we conducted a survey experiment on a fresh sample of 820 respondents. ${ }^{24}$ In this second survey, roughly half of the respondents $(n=401)$ first answered a ten-item fixed battery. ${ }^{25}$ The remaining respondents $(n=419)$ answered ten items as selected by the CAT algorithm discussed above. Respondents in both groups then answered the remaining 54 items presented in a random order.

Requiring all respondents to complete the entire battery allows us to evaluate the two measurement techniques using a common metric-respondents' scores as assessed by the complete 64-item battery. Thus, we approximated respondent $j$ 's true latent trait value, $\theta_{j}$, using her answers to the 64 questions. We then computed estimated values of $\hat{\theta}_{j}^{\text {(EAP) }}$ based on the first $n \in(3,5,7,10)$ questions administered in either treatment condition. ${ }^{26}$ Our purpose is to evaluate how well $\hat{\theta}_{j}^{\text {(EAP) }}$ approximates $\theta_{j}$ across treatment conditions.

Note that the data from the calibration sample are used only for item selection. That is, we reestimated the entire measurement model using only the fresh sample. This provides a fair test of the method, as we are not simply assuming that the model estimated on the calibration sample is true for the second (experimental) sample.

Figure 4 shows the squared error of the estimated values of $\hat{\theta}_{j}^{(\mathrm{EAP})}$ for individuals in the dynamic (squares) and static (triangles) treatment conditions. The lines represent loess curves for each population. ${ }^{27}$ As in the simulations in Section 4, the dynamic algorithm outperforms the fixed battery for all values of $\theta_{j}$ and the difference is particularly noticeable for larger values of $n$ and more extreme values of $\theta_{j}{ }^{28}$

Figure 5 compares the static and dynamic scales of various lengths at the population level. The upper-left panel shows the median squared error (MSE) while the upper-right panel shows the median absolute bias, measured as $\left|\theta_{j}-\hat{\theta}_{j}^{(\mathrm{EAP})}\right|$. Likewise, the lower-left panel shows the total absolute bias for each of the samples, and the lower-right panel shows the median posterior variance for the estimates. The figure shows that CAT offers dramatic improvements in measurement accuracy relative to static scales on each of these metrics. The measures are both less biased and more precisely estimated. Indeed, by these metrics a CAT scale with only six items outperforms a ten-item fixed scale. Extrapolating from this example, Fig. 5 suggests that CAT offers the potential for $40 \%$ reduction in battery length on surveys with no loss in measurement quality.

Finally, the increased accuracy and precision resulting from using the adaptive measure affects the inferences we draw from the data. ${ }^{29}$ To begin with, the dynamic battery is able to provide a more fine-grained measure that better reflects heterogeneity within the sample. For instance, the estimated sample variance on the political knowledge scale is only 0.65 when using a five-item static battery, while it is $15 \%$ greater $(0.75)$ for the sample that took the dynamic battery. Likewise, fully $21 \%$ of respondents taking the five-item static scale were placed in the most extreme category as either the most or least knowledgeable respondents in the sample. However, only $5 \%$ of

\footnotetext{
${ }^{24}$ Respondents were again based in the United States and over the age of 18 years and were recruited using Amazon Mechanical Turk. Respondents who had participated in the first-round survey were excluded from this analysis.

${ }^{25} \mathrm{We}$ designed the fixed battery to provide 3-item, 5-item, 7-item, and 10-item batteries with good measurement properties. That is, we chose items that spanned the range of difficulty and had relatively large discrimination parameters in the calibration sample. Moreover, in choosing between similarly performing items, we selected questions most similar to the standard ANES measure of political knowledge (e.g., "Who is the Speaker of the House of Representatives?"). The items in the fixed battery are indicated in Table 2.

${ }^{26}$ Observe that, because the CAT algorithm minimizes $\hat{\theta}_{j}^{(\mathrm{EAP})}$ at every step of the test, we can compare the results obtained by stopping at any given point without loss of generality.

${ }^{27}$ We used a two-sided Wilcoxon test to determine whether the distributions were indeed statistically different. The results of the test confirm what we observed visually. That is, squared error is significantly lower in all cases with $p<0.001$. The rank-sum statistics were $W=69470,43790,28108$, and 13546 for $n=3,5,7$, and 10, respectively.

${ }^{28}$ Some caution is needed in comparing the performance of the two methods for the extreme values of $\theta$ due to the increasingly small sample size.

${ }^{29}$ We thank an anonymous reviewer for suggesting this analysis.
} 


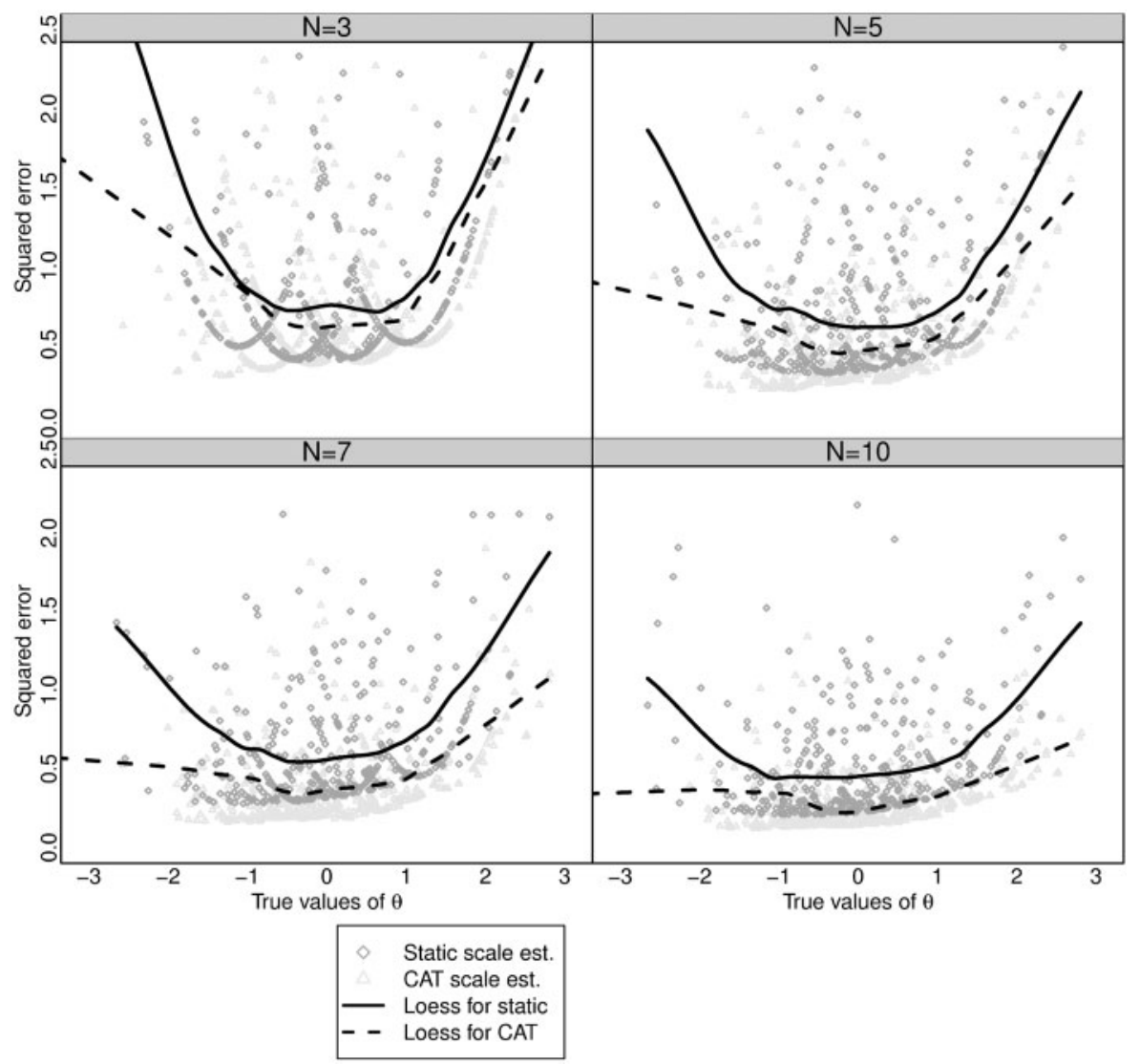

Fig. 4 Out-of-sample squared error for dynamic and static scales of four different lengths. The points show the squared error, defined as $\operatorname{Var}\left(\hat{\theta}_{j}^{(\mathrm{EAP})}\right)+\left(\theta_{j}-\hat{\theta}_{j}^{(\mathrm{EAP})}\right)^{2}$, for each individual using both the CAT (triangles) and static (circles) batteries of length 3, 5, 7, and 10. The lines are loess estimates for each method.

respondents taking a dynamic scale of an identical length were so categorized due to the adaptive nature of the CAT battery.

The increased precision and reduced bias also improve the external validity of the knowledge measure. We can see this by examining the level of correlation between our political knowledge measure and other responses that we would expect to be correlated with political knowledge. Table 3 shows bivariate regressions for the five-item dynamic and static political knowledge measures. The "outcomes" in these regressions are answers to three additional survey items: the respondents' level of interest in politics (a four-point scale), the frequency with which they discuss politics and current affairs (a seven-point scale), and the degree to which they report paying attention to national and international issues (a seven-point scale). ${ }^{30}$

To make the coefficients comparable, we re-scaled the knowledge batteries to range between 0 and 1. Table 3 shows that the coefficients for the dynamic political knowledge scale are always larger. Moreover, the differences are substantive. For example, our political interest questions ask the respondent, "How interested would you say you are in politics and current affairs: Not at all interested; Not very interested; Somewhat interested; or Very interested?" Moving from the minimum to the maximum of political knowledge as measured by the static five-item knowledge battery is associated with an average change of 1.31 on this scale. However, because of the improved measurement properties of the five-item adaptive battery, moving from a minimum to

$\overline{{ }^{30} \text { All survey question }}$ wording and response options are shown in the online supplemental materials. 
Median squared error

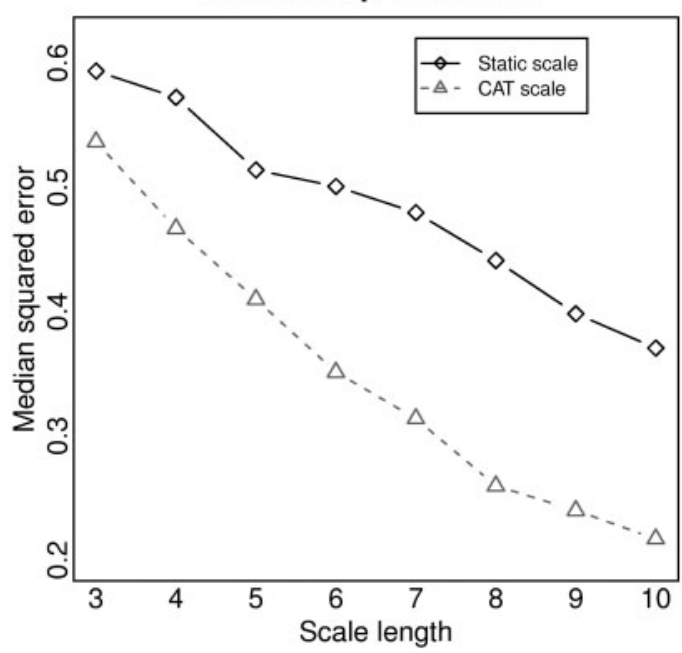

Total absolute bias

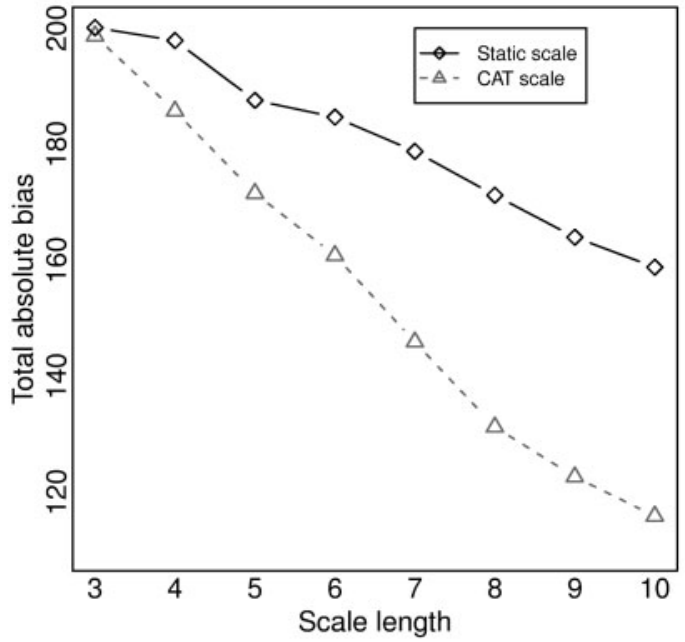

Median absolute bias

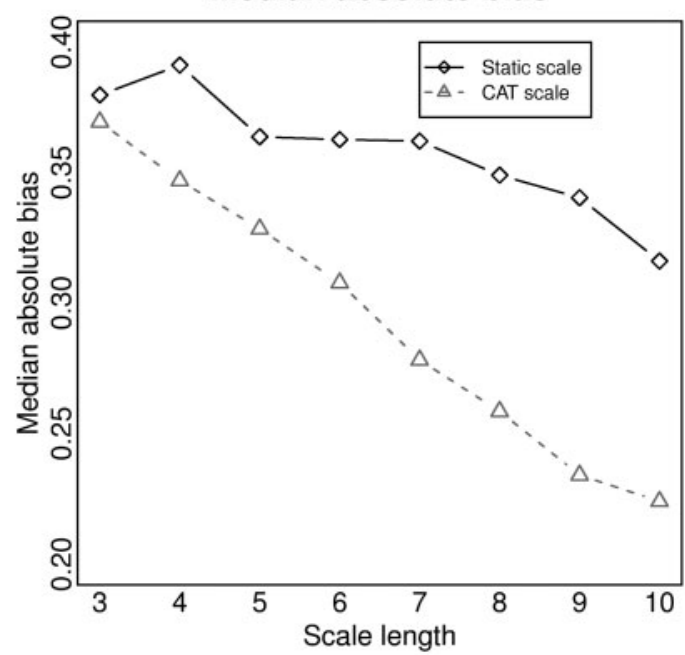

Median posterior variance

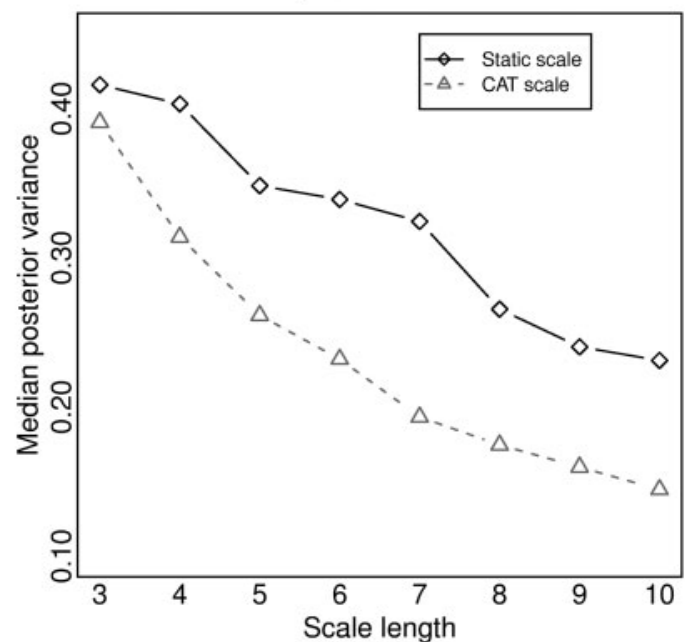

Fig. 5 Comparing measurement quality by battery length. The points in the upper-left panel show the median squared error for the sample individual using both the CAT (light triangles) and static (dark squares) batteries of various lengths. The points on the upper-right panel show the median absolute bias, defined as $\left|\theta_{j}-\hat{\theta}_{j}^{\text {(EAP) }}\right|$. The points on the lower-left panel show the total absolute bias for the sample, while the lower-right panel shows the median posterior variance for the estimates.

a maximum level of political knowledge is associated with a 2.08-unit change for respondents randomly assigned to answer the dynamic battery. Table 3 shows similar differences for all three outcomes.

\section{Conclusion}

While we believe the evidence presented above suggests that CAT offers a superior approach to traditional static batteries, the methodology comes with several caveats and limitations that are important to note. First, CAT is only appropriate when researchers are interested in placing respondents on some latent scale rather than examining responses to specific questions. Second, CAT should not be used for batteries where there is evidence of strong question-order effects.

Third, CAT requires pre-testing of battery items to calibrate the model. Although pre-testing of items is generally considered ideal for public opinion research, it is not always feasible. This 
Table 3 Comparing external validity of five-item dynamic and static political knowledge batteries

\begin{tabular}{|c|c|c|c|c|c|c|}
\hline & \multicolumn{2}{|c|}{ Interest in politics } & \multicolumn{2}{|c|}{ Frequently discuss } & \multicolumn{2}{|c|}{ Attention to politics } \\
\hline & Dyn. & Static & Dyn. & Static & Dyn. & Static \\
\hline Constant & $3.34(0.11)$ & $2.78(0.08)$ & $5.58(0.23)$ & $4.92(0.16)$ & $4.58(0.21)$ & $3.56(0.14)$ \\
\hline Knowledge & $2.08(0.17)$ & $1.31(0.13)$ & $3.45(0.35)$ & $2.78(0.25)$ & $3.42(0.32)$ & $2.07(0.23)$ \\
\hline$R^{2}$ & 0.26 & 0.20 & 0.19 & 0.23 & 0.21 & 0.17 \\
\hline$n$ & 418 & 400 & 418 & 401 & 418 & 401 \\
\hline
\end{tabular}

Note. To make the coefficients comparable, the knowledge scores are re-scaled so that a one-unit change represents moving from the minimum to the maximum observed value in each sample. Note that the coefficients are always substantially larger for the dynamic battery, indicating higher levels of correlation. All survey question wording and response options are shown in the online supplementary materials.

suggests that there may be a trade-off for the costs of reducing the length of batteries and the costs of pre-testing batteries. The more accurate the pre-test (i.e., the larger the sample size and the more representative the sample), the greater the potential for reducing battery length while preserving measurement accuracy.

In part, pre-testing costs may be ameliorated by making survey data and item calibrations widely available to other researchers, thus sharing the costs of pre-testing. In any case, we note that even the selection of static reduced batteries is based on some kind of pre-test data or prior understanding. We strongly believe that, ceteris paribus, CAT algorithms will always outperform static batteries for any fixed level of pre-test information or prior beliefs about item performance.

Finally, for time-varying attitudes or traits, the calibration may not always remain current or appropriate. This is not an issue for measurement of traits like personality, but could be problematic for less stable attitudes like presidential approval. Further research is needed to develop methods that can detect when specific item parameters have become obsolete (e.g., Segall 2002).

We conclude by noting several promising paths forward for this research. While numerous variations in CAT algorithms are available, the examples in this article implemented only uninformative priors, MEPV item selection, EAP ability estimation, and fixed-length batteries. Future research could explore which algorithms of the many available in the literature are most appropriate for various types of researcher constraints, whether they be time, cost, or measurement precision. Additional guidance as to the relative advantages and disadvantages of various CAT approaches may facilitate wider adoption of the methodology.

Furthermore, this article restricted itself to dichotomous data. While this is useful for many political science applications, there are also numerous latent traits that are more appropriately measured using polytomous models. Though the intuition behind such models is similar to that described above, implementation issues remain. Moreover, it may be that CAT offers limited advantages for Likert-type survey items relative to static batteries. Future studies should investigate the benefits of CAT surveys for ordered-categorical survey questions.

Finally, we note that there are several extensions to CAT algorithms that may significantly improve performance beyond what we show here. These include the development of informative priors based on earlier survey response (van der Linden 1999) and accounting for response times (van der Linden 2008). In addition, we believe that additional research is warranted on the development of priors more appropriate for survey research because the battery size is likely to be quite short and responses may include more error relative to educational testing applications.

Although there is room for continued improvement and extension, we have shown in this article that CAT techniques are capable of obviating the need for public opinion researchers to choose between administering large multi-item scales or selecting a single reduced scale to administer to all respondents, which may reduce measurement quality. Adaptive testing allows for the administration of fewer questions while achieving superior levels of statistical precision and accuracy relative to any static reduced scale. We believe that CAT may provide substantial cost savings and efficiency gains for survey researchers while reducing attrition and nonresponse. 
After presenting the details of one CAT algorithm, we demonstrated the method using both simulation and an empirical example. Using a battery of political knowledge items, we administered a set of 64 questions to 810 respondents and calibrated the CAT algorithm on their responses. When compared to a fixed battery, CAT provided both improved measurement precision and accuracy for a fresh sample of 820 respondents. This was particularly true for larger numbers of questions and more "extreme" respondents. Finally, we have developed software to administer such dynamic surveys. This software will be made available to researchers who wish to adopt CAT techniques using a variety of survey platforms. ${ }^{31}$

\section{Funding}

This work was supported by grants from the Weidenbaum Center on the Economy, Government, and Public Policy at Washington University in Saint Louis and the National Science Foundation [SES-1023762 to J.M.M.].

\section{References}

Anderson, A., A. Basilevsky, and D. Hum. 1983. Missing data: A review of the literature. In Handboook of survey research, eds. P. H. Rossi, J. D. Wright, and A. B. Anderson, 415-81. New York: Academic Press.

Bafumi, J., A. Gelman, D. K. Park, and N. Kaplan. 2005. Practical issues in implementing and understanding Bayesian ideal point estimation. Political Analysis 13(2):171-87.

Bafumi, J., and M. C. Herron. 2010. Leapfrog representation and extremism: A study of American voters and their members in Congress. American Political Science Review 104(3):519-42.

Bailey, M. A. 2007. Comparable preference estimates across time and institutions for the court, Congress, and presidency. American Journal of Political Science 51(3):433-48.

Baker, F. B., and S.-H. Kim. 2004. Item response theory: Parameter estimation techniques. New York: Marcel Dekker.

Barabas, J. 2002. Another look at the measurement of political knowledge. Political Analysis 10(2):209-22.

Berinsky, A. J., G. A. Huber, and G. S. Lenz. 2012. Evaluating online labor markets for experimental research: Amazon.com's Mechanical Turk. Political Analysis 20(3):329-50.

Brewer, P. R. 2003. Values, political knowledge, and public opinion about gay rights. Public Opinion Quarterly 67(3):173-201.

Burchell, B., and C. Marsh. 1992. The effect of questionnaire length on survey response. Quality \& Quantity 26(3):233-44.

Cacioppo, J. T., and R. E. Petty. 1984. The efficient assessment of need for cognition. Journal of Personality Assessment 48(3):306-7.

Choi, S. W., and R. J. Swartz. 2009. Comparison of CAT item selection criteria for polytomous items. Applied Psychological Measurement 33(6):419-40.

Clinton, J. D., and A. Meirowitz. 2001. Agenda constrained legislator ideal points and the spatial voting model. Political Analysis 9(3):242-59.

2003. Integrating voting theory and roll call analysis: A framework. Political Analysis 11(4):381-96.

Clinton, J., S. Jackman, and D. Rivers. 2004. The statistical analysis of roll call voting: A unified approach. American Political Science Review 98(2):355-70.

Crawford, S. D., M. P. Couper, and M. J. Lamias. 2001. Web surveys: Perceptions of burden. Social Science Computer Review 19(2):146-62.

DeBell, M. 2012. Harder than it looks: Coding political knowledge on the ANES. Paper presented at the 2012 meeting of the Midwest Political Science Association, Chicago, IL.

Delli Carpini, M. X., and S. Keeter. 1993. Measuring political knowledge: Putting first things first. American Journal of Political Science 37(4):1179-206. 1996. What Americans know about politics and why it matters. New Haven, CT: Yale University Press.

Dodd, B. G., R. De Ayala, and W. R. Koch. 1995. Computerized adaptive testing with polytomous items. Applied Psychological Measurement 19(1):5-22.

Embretson, S. E., and S. P. Reise. 2000. Item response theory for psychologists. Mahwah, NJ: Lawrence Erlbaum.

Feldman, S., and L. Huddy. 2005. Racial resentment and white opposition to race-conscious programs: Principles or prejudice? American Journal of Political Science 49(1):168-83.

Forbey, J. D., and Y. S. Ben-Porath. 2007. Computerized adaptive personality testing: A review and illustration with the MMPI-2 computerized adaptive version. Psychological Assessment 19(1):14-24.

Galesic, M., and M. Bosnjak. 2009. Effects of questionnaire length on participation and indicators of response quality in Web surveys. Public Opinion Quarterly 73(2):349-60.

\footnotetext{
${ }^{31}$ All data used to generate the results in this article will be made available to the public in the journal's dataverse upon publication at http://hdl.handle.net/1902.1/19381 (Montgomery and Cutler 2012).
} 
Gerber, A. S., G. A. Huber, D. Doherty, C. M. Dowling, and S. E. Ha. 2010. Personality and political attitudes: Relationships across issue domains and political contexts. American Political Science Review 104(01):111-33.

Gibson, J. L., and G. A. Caldeira. 2009. Knowing the Supreme Court? A reconsideration of public ignorance of the high court. Journal of Politics 71(2):429-41.

Gillion, D. Q. 2012. Re-defining political participation through item response theory. Unpublished paper.

Gomez, B. T., and J. M. Wilson. 2001. Political sophistication and economic voting in the American electorate: A theory of heterogeneous attribution. American Journal of Political Science 45(4):899-914.

Gosling, S. D., P. J. Rentfrow, and W. B. Swann. 2003. A very brief measure of the big-five personality domains. Journal of Research in Personality 37(6):504-28.

Heberlein, T. A., and R. Baumgartner. 1978. Factors affecting response rates to mailed questionnaires: A quantitative analysis of the published literature. American Sociological Review 43(4):447-62.

Herzog, A. R., and J. G. Bachman. 1981. Effects of questionnaire length on response quality. Public Opinion Quarterly 45(4):549-59.

Hol, A. M., H. C. Vorst, and G. J. Mellenbergh. 2007. Computerized adaptive testing for polytomous motivation items: Administration mode effects and a comparison with short forms. Applied Psychological Measurement 31(5):412-29.

Jackman, S. 2001. Multidimensional analysis of roll call data via Bayesian simulation: Identification, estimation, inference, and model checking. Political Analysis 9(3):227-41.

Kingsbury, G., and D. J. Weiss. 1983. A comparison of IRT-based adaptive mastery testing and a sequential mastery testing procedure. In New horizons in testing: Latent trait test theory and computerized adaptive testing, ed. D. J. Weiss. New York: Academic Press.

Krosnick, J. A. 1991. Response strategies for coping with the cognitive demands of attitude measures in surveys. Applied Cognitive Psychology 5(3):213-36.

- 1999. Survey research. Annual Review of Psychology 50:537-67.

Krosnick, J. A., A. L. Holbrook, M. K. Berent, R. A. B. T. Carson, W. Hanemann, R. J. Kopp, C. Mitchell, R. Cameron, S. Presser, P. A. Ruud, V. Smith, W. R. Moody, M. C. Green, and M. Conaway. 2002. The impact of "no opinion" response options on data quality: Non-attitude reduction or an invitation to satisfice? Public Opinion Quarterly 66(3): 371-403.

Lord, F. M. 1980. Applications of item response theory to practical testing problems. Hillsdale, NJ: L. Erlbaum Associates.

Lord, F., and M. R. Novick. 1968. Statistical theories of mental test scores. Reading, MA: Addison-Wesley.

Lupia, A. 2006. How elitism undermines the study of voter competence. Critical Review 18(1-3):217-32.

. 2008. Procedural transparency and the credibility of election surveys. Electoral Studies 27(4):732-9.

Luskin, R. C. 1987. Measuring political sophistication. American Journal of Political Science 31(4):856-99.

Luskin, R. C., and J. G. Bullock. 2011. "Don't know" means "don't know": DK responses and the public's level of political knowledge. Journal of Politics 73(2):547-57.

Martin, A. D., and K. M. Quinn. 2002. Dynamic ideal point estimation via Markov chain Monte Carlo for the US Supreme Court, 1953-1999. Political Analysis 10(2):134-53.

Matthews, R. A., L. M. Kath, and J. L. Barnes-Farrell. 2010. A short, valid, predictive measure of work-family conflict: Item selection and scale validation. Journal of Occupational Health Psychology 15(1):75-90.

Mondak, J. J., and M. R. Anderson. 2004. The knowledge gap: A reexamination of gender-based differences in political knowledge. Journal of Politics 66(2):492-512.

Mondak, J. J., and B. C. Davis. 2001. Asked and answered: Knowledge levels when we will not take "don't know" for an answer. Political Behavior 23(3):199-224.

Mondak, J. J. 2001. Developing valid knowledge scales. American Journal of Political Science 45(1):224-38.

Montgomery, J. M., and J. Cutler. 2012. Replication data for: Computerized adaptive testing for public opinion surveys. http://hdl.handle.net/1902.1/19381 IQSS Dataverse Network.

Piazza, T., P. M. Sniderman, and P. E. Tetlock. 1989. Analysis of the dynamics of political reasoning: A general-purpose computer-assisted methodology. Political Analysis 1(1):99-119.

Podsakoff, P. M., and S. B. MacKenzie. 1994. An examination of the psychometric properties and nomological validity of some revised and reduced substitutes for leadership scales. Journal of Applied Psychology 79(5):702-13.

Poole, K. T. 2005. Spatial models of parliamentary voting. New York: Cambridge University Press.

Prior, M., and A. Lupia. 2008. Money, time, and political knowledge: Distinguishing quick recall and political learning skills. American Journal of Political Science 52(1):19-183.

Prior, M. 2012. Visual political knowledge: A different road to competence. Unpublished paper.

Richins, M. L. 2004. The material values scale: Measurement properties and development of a short form. Journal of Consumer Research 31(1):209-19.

Russell, S. S., C. Spitzmüller, L. F. Lin, J. M. Stanton, P. C. Smith, and G. H. Ironson. 2004. Shorter can also be better: The abridged job in general scale. Educational and Psychological Measurement 64(5):878-93.

Segall, D. O. 2002. Confirmatory item factor analysis using Markov chain Monte Carlo estimation with applications to online calibration in CAT. Paper presented at the annual meeting of the National Council on Measurement in Education, New Orleans, LA.

. 2005. Computerized adaptive testing. In Encyclopedia of social measurement, ed. K. Kempf-Leonard, Vol. 1, 429-38. Oxford, UK: Elsevier. 
2010. Principles of multidemensional adaptive testing. In Elements of adaptive testing, eds. W. J. van der Linden and C. A. W. Glas, 57-76. New York: Springer.

Sheatsley, P. 1983. Questionnaire construction and item writing. In Handboook of survey research, eds. P. H. Rossi, J. D. Wright, and A. B. Anderson, 195-230. New York: Academic Press.

Singh, J., R. D. Howell, and G. K. Rhoads. 2007. Designs for Likert-type data: An approach for implementing marketing surveys. Journal of Marketing Research 19(1):12-24.

Sniderman, P. M., R. A. Brody, and P. E. Tetlock. 1991. Reasoning and choice: Explorations in political psychology. New York: Cambridge University Press.

Sniderman, P. M., T. Piazza, P. E. Tetlock, and A. Kendrick. 1991. The new racism. American Journal of Political Science 35(2):423-47.

Stanton, J. M., E. F. Sinar, W. K. Balzer, and P. C. Smith. 2002. Issues and strategies for reducing the length of selfreport scales. Personnel Psychology 55(1):167-94.

Thompson, E. R. 2012. A brief index of affective job satisfaction. Group \& Organization Management 37(3):275-307.

Treier, S., and D. S. Hillygus. 2009. The nature of political ideology in the contemporary electorate. Public Opinion Quarterly 73(4):679-703.

Treier, S., and S. Jackman. 2008. Democracy as a latent variable. American Journal of Political Science 52(1):201-17.

van der Linden, W. J. 1998. Bayesian item selection criteria for adaptive testing. Psychometrika 63(2):201-16.

. 1999. Empirical initialization of the trait estimator in adaptive testing. Applied Psychological Measurement 23(1):21-29.

. 2008. Using response times for item selection in adaptive testing. Journal of Educational and Behavioral Statistics 33(1):5-20.

2010. Constrained adaptive testing with shadow tests. In Elements of adaptive testing, eds. W. J. van der Linden and C. A. W. Glas, 31-56. New York: Springer.

van der Linden, W. J., and P. J. Pashley. 2010. Elements of adaptive testing. New York: Springer.

Verba, S., K. L. Schlozman, and H. E. Brady. 1995. Voice and equality: Civic voluntarism in American politics. Cambridge, MA: Harvard University Press.

Wainer, H. 1990. Introduction and history. In Computerized Adaptive Testing: A Primer, ed. H. Wainer. Hillsdale, NJ: Lawrence Erlbaum Associates.

Waller, N. G., and S. P. Reise. 1989. Computerized adaptive personality assessment: An illustration with the absorption scale. Journal of Personality and Social Psychology 57(6):1051.

Weiss, D. J. 1982. Improving measurement quality and efficiency with adaptive testing. Applied Psychological Measurement 6(4):473-92.

Weiss, D. J., and G. G. Kingsbury. 1984. Application of computerized adaptive testing to educational problems. Journal of Educational Measurement 21(4):361-75.

$\mathrm{Xu}, \mathrm{X}$., and J. Douglas. 2006. Computerized adaptive testing under nonparamteric IRT models. Psychometrika 71(1):121-37.

Yammarino, F. J., S. J. Skinner, and T. L. Childers. 1991. Understanding mail survey response behavior: A metaanalysis. Public Opinion Quarterly 55(4):613-639.

Zaller, J. R. 1992. The nature and origins of mass opinion. New York: Cambridge University Press. 